\title{
Scar formation after lower eyelid incision for reconstruction of the inferior orbital wall related to the lower eyelid crease or ridge in Asians
}

Seong Jin Oh,

Kwang Seog Kim,

Jun Ho Choi,

Jae Ha Hwang,

Sam Yong Lee

Department of Plastic and

Reconstructive Surgery, Chonnam

National University Medical School,

Gwangju, Korea

\begin{abstract}
Background: Transcutaneous lower eyelid approaches are associated with a risk of postoperative scarring depending on the distance between the incision line and the lower eyelid margin. The lower eyelid crease of Caucasians corresponds to a ridge-shaped fold in young Asians. However, this relationship has not been sufficiently evaluated in the latter. The authors, therefore, investigated the location of the scar and the lower eyelid crease or ridge to find the optimal location for the incision line.

Methods: This study included 60 out of 139 patients who underwent inferior orbital wall reconstruction through a lower eyelid skin incision between July 2019 and June 2020. According to the location of the scar, the patients were classified into three groups: group $A$ ( $\geq 2 \mathrm{~mm}$ above the lower eyelid crease or ridge), group $B$ (within the lower eyelid crease or ridge to $2 \mathrm{~mm}$ above the lower eyelid crease or ridge), and group C (within the lower eyelid crease or ridge to $2 \mathrm{~mm}$ below the lower eyelid crease or ridge). At 6 or 12 months after surgery, the Patient and Observer Scar Assessment Scale (POSAS) score was obtained, the distance between the lower eyelid margin and the scar (DMS) and the distance between the margins of the peripheral pupil and the lower eyelid (DMPE) were measured, and the occurrence of ectropion was evaluated.

Results: Group B had the lowest POSAS score (A: $22.7 \pm 8.0$, B: $20.9 \pm 2.4$, C: $32.5 \pm 4.1, p<0.001$ ). Linear regression analysis showed that the DMS was positively correlated with the POSAS score $(p<0.001)$ and that the risk of DMPE widening increased as the DMS decreased $(p=0.029)$. None of the patients had ectropion.

Conclusion: When using the transcutaneous approach for inferior orbital wall reconstruction, the optimal incision site is within the lower eyelid crease or ridge to $2 \mathrm{~mm}$ above the lower eyelid crease or ridge.
\end{abstract}

Abbreviations: DMCR, distance between the lower eyelid margin and the lower eyelid crease or ridge; DMPE, distance between the margins of the peripheral pupil and the lower eyelid; DMS, distance between the lower eyelid margin and the scar; POSAS, Patient and Observer Scar Assessment Scale

Keywords: Ectropion / Eyelid / Facial bone / Scar

\footnotetext{
Correspondence: Kwang Seog Kim

Department of Plastic and Reconstructive Surgery, Chonnam National University Medical School, 42 Jebong-ro, Dong-gu, Gwangju 61469, Korea

E-mail: pskim@chonnam.ac.kr

This article is a master's thesis of Seong Jin Oh submitted in 2021 at Chonnam National University Medical School.

Received October 14, 2021 / Revised November 15, 2021 / Accepted December 9, 2021
}

\section{INTRODUCTION}

Inferior orbital wall fractures are very common, and often caused by traffic accidents and sports in modern society [1,2]. 
In inferior orbital wall fractures, the treatment of choice is open reduction and internal fixation for improving ocular symptoms and maintaining facial symmetry $[3,4]$. The inferior orbital wall can be accessed by a transconjunctival or a transcutaneous approach. Depending on the incision, the transcutaneous approach can be classified into three types: subciliary, subtarsal, and infraorbital. It can also be divided into skin flap, nonstepped skin-muscle flap, and stepped skin-muscle flap methods, depending on the dissection technique $[5,6]$.

The site and technique of a lower eyelid incision affect accessibility, extent of exposure, postoperative scarring, and risk of complications, thereby greatly influencing surgical outcomes. For this reason, it is of paramount importance to determine which approach is the most appropriate, on the basis of the purposes of surgery and the condition of the patient's lower eyelid $[7,8]$. Although studies comparing and analyzing various approaches have been actively conducted, the ideal approach for inferior orbital wall reconstruction has not yet been established. In addition, the incision site, in most studies, has been determined on the basis of the approximate distance from the lower eyelid margin during the transcutaneous approach. Few studies have, furthermore, compared the results of various incisions using the transcutaneous approach in Asians. Firstly, the ideal approximate distance chosen by each surgeon differs in many cases. Secondly, making an incision according to the approximate distance alone is neglectful of anatomical differences among individual patients, including variations according to ethnicity, sex, and age.

In Caucasians, the lower eyelid crease is a natural skin fold formed according to the structure of the capsulopalpebral fascia and lower eyelid skin. However, in young Asians, the lower eyelid crease presents as a ridge-shaped fold because of the anatomical structure of the high fusion of the capsulopalpebral fas- cia and orbital septum, and projected fat tissue around the inferior margin of the lower eyelid tarsus [9-11]. The authors named this fold the "lower eyelid ridge" (Fig. 1). The purpose of this study was to investigate whether the lower eyelid crease or ridge can be used as a reference point to determine the ideal position of the lower eyelid incision in the reconstruction of the inferior orbital wall in Asians resulting in the most inconspicuous scar.

\section{METHODS}

\section{Participants}

From July 2019 to June 2020, 139 patients underwent inferior orbital wall reconstruction through a lower eyelid incision at our hospital. The exclusion criteria of this study were as follows: (1) patients who underwent surgery on the orbital rim or orbital floor of both eyes and (2) patients who had an accompanying (a) soft tissue injury in the lower eyelid at the time of trauma, (b) infection or hematoma during the recovery process after surgery, (c) facial bone asymmetry, and (d) systemic diseases that could affect wound healing (e.g., long-term steroid use and immunosuppression). Of 139 patients, 60 who met the criteria were prospectively investigated in the study. The snap back test and lid distraction test were performed preoperatively. The incision site was estimated based on the patients' postoperative scars. In general, the locations of the subciliary incision $(2 \mathrm{~mm})$ and subtarsal incision $(5-7 \mathrm{~mm}$ ) were determined based on the approximate distance from the lower eyelid margin. The authors classified the patients based on this approximate distance. According to the location of the scar, the patients were classified into three groups: group A ( $\geq 2 \mathrm{~mm}$ above the lower eyelid crease or ridge), group $B$ (within the lower eyelid crease or ridge to $2 \mathrm{~mm}$ above the lower eyelid crease or ridge), and
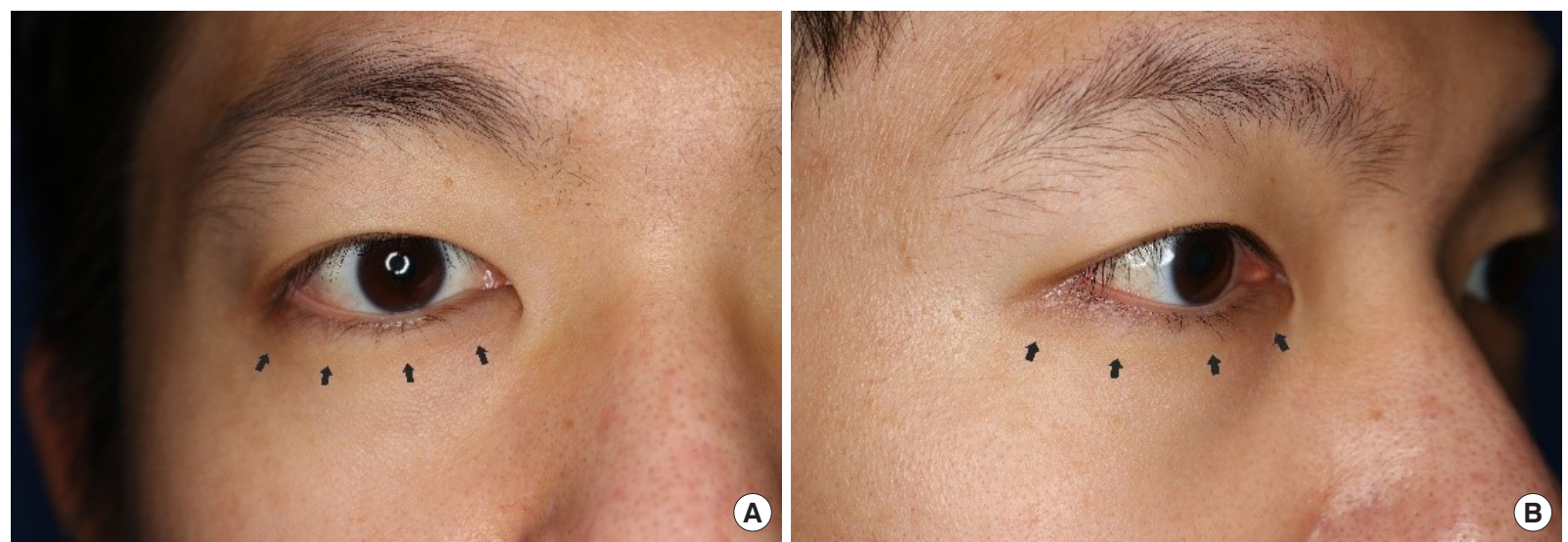

Fig. 1. Lower eyelid ridge in a young Asian. Thin pretarsal skin and thick preseptal skin form a natural ridge (black arrows) at a level similar to that of the lower eyelid crease in Caucasians. (A) Frontal view. (B) Oblique view. 
group C (within the lower eyelid crease or ridge to $2 \mathrm{~mm}$ below the lower eyelid crease or ridge) (Fig. 2). Patients were followed up at 6 months or 12 months after surgery and evaluated.

\section{Surgical technique}

Under general anesthesia, an incision line was designed on the lower eyelid. The skin was incised after injection of lidocaine mixed with epinephrine at a ratio of 1:100,000. The stepped skin-muscle flap dissection method was used to reach the inferior orbital rim in all patients: a skin flap with a 2-3 mm width was elevated from the incision line in the distal direction. Next, a myocutaneous flap was lifted through the orbicularis muscle up to the orbital rim, and an incision was made on the periosteum of the orbital rim to access the fracture site. An absorbable micro-C-plate with $0.5 \mathrm{~mm}$ thickness was used for the orbital rim, and an absorbable mesh plate with the same thickness was used for the orbital floor in all patients. After reduction and rigid fixation using a plate and screws, the periosteum, orbicularis muscle, and skin were closed layer by layer. Tarsal strip anchoring was not performed in any patient.

\section{Data collection}

Data were collected on the patients' age, fracture type, surgery type, and fracture location. The patients visited our outpatient clinic at 6 months or 12 months after surgery, and were evaluated by two plastic surgeons. The value of each test was recorded as the average of those obtained by the two evaluators. The Patient and Observer Scar Assessment Scale (POSAS) [12] was used to assess the scar quality. The distance between the lower

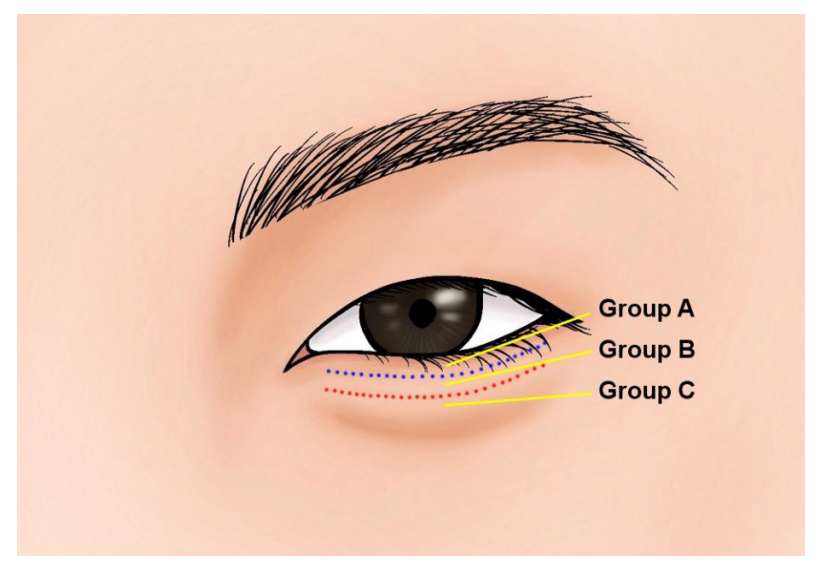

Fig. 2. Study groups according to the location of the scar. Group A contains patients whose scar is located above the blue line. Group B comprises patients whose scar is located between the blue and red lines. Group C includes patients whose scar is located between the red line and $2 \mathrm{~mm}$ below the lower eyelid crease or ridge. Red line, lower eyelid crease or ridge; blue line, a virtual line $2 \mathrm{~mm}$ above the lower eyelid crease or ridge. eyelid margin and the scar (DMS) and the distance between the lower eyelid margin and the lower eyelid crease or ridge (DMCR) of both sides were measured at the mid-pupillary line with the patient sitting upright and the eyes in forward gaze. The distance between the margins of the peripheral pupil and the lower eyelid (DMPE) was measured under the same light source in the same position (Fig. 3). DMPE widening was considered to be present if there was a difference of $\geq 1 \mathrm{~mm}$ between the operated and the unoperated sides. The occurrence of ectropion and the presence of the lateral extension scar were investigated.

\section{Statistical analysis}

The subjects' characteristics according to the study group were presented as mean \pm standard deviation or number (\%). A oneway analysis of variance with Scheffe's post-hoc test and Fisher exact test was conducted to estimate the significance of differences among the study groups. To assess the differences in the POSAS subscale according to the study group, a one-way analysis of variance was conducted. Simple and multivariate linear regressions were performed to evaluate the association between the POSAS score and the DMS. Simple and multivariate logistic regression analyses were conducted to identify the association between DMS and DMPE widening. All multivariate models included lateral extension, the time of scar evaluation, and age. Statistical significance was set at $p<0.05$. All analyses were performed using R version 4.0.2 (R Foundation for Statistical Computing, Vienna, Austria).

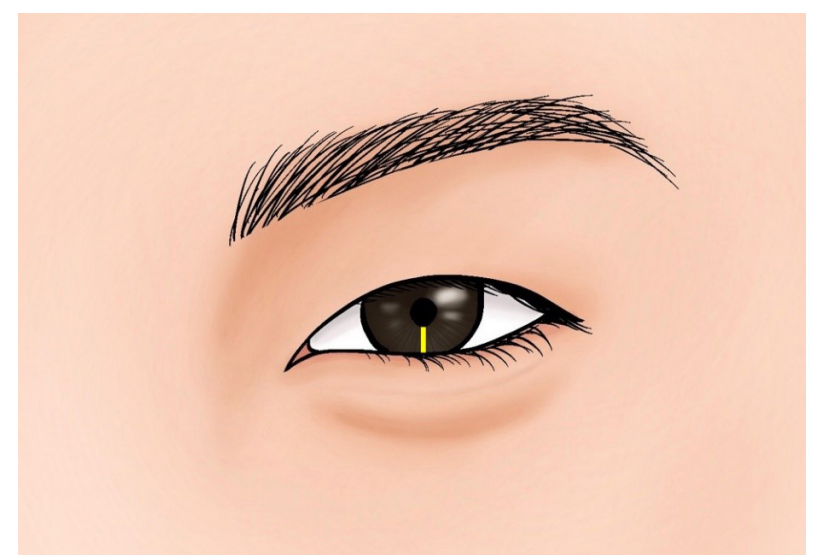

Fig. 3. Distance between the margins of the peripheral pupil and the lower eyelid (DMPE). The DMPE (yellow line) is checked by comparing this distance with that of the opposite eye. DMPE widening is defined as a difference between the two distances of more than $1 \mathrm{~mm}$. 


\section{RESULTS}

\section{Patient demographics}

\section{Study groups}

In total, 139 patients underwent inferior orbital wall reconstruction through a lower eyelid incision. Thirty-one patients were excluded owing to loss on follow-up. Thirteen patients who underwent surgery on both eyes were excluded. Thirtythree patients with accompanying soft tissue injury in the lower eyelid at the time of trauma and two patients who developed hematoma during the recovery process after surgery, were also excluded because of the possibility that the lesions could affect the wound healing and scarring process. No patient developed

Table 1. Summary of patient data

\begin{tabular}{|c|c|c|c|c|}
\hline Variable & $\begin{array}{l}\text { Group A } \\
(n=23)\end{array}$ & $\begin{array}{l}\text { Group B } \\
(n=24)\end{array}$ & $\begin{array}{l}\text { Group C } \\
(n=13)\end{array}$ & $p$-value \\
\hline Age (yr) & $43.2 \pm 17.2$ & $41.8 \pm 13.8$ & $34.1 \pm 17.3$ & 0.127 \\
\hline Fracture type & & & & 0.311 \\
\hline Tripod fracture & $16(69.6)$ & 14 (58.3) & $9(69.2)$ & \\
\hline Inferior orbital wall fracture & $3(13.0)$ & 8 (33.3) & $4(30.8)$ & \\
\hline Both & $4(17.4)$ & $2(8.3)$ & 0 & \\
\hline Fracture site & & & & 0.918 \\
\hline Medial & $4(17.4)$ & $5(20.8)$ & $4(30.8)$ & \\
\hline Central & $14(60.9)$ & $15(62.5)$ & $7(53.8)$ & \\
\hline Lateral & $5(21.7)$ & $4(16.7)$ & $2(15.4)$ & \\
\hline POSAS & $22.7 \pm 8.0$ & $20.9 \pm 2.4$ & $32.5 \pm 4.1$ & $<0.001^{\mathrm{a}}$ \\
\hline Group A:Group B & & & & 0.548 \\
\hline Group B:Group C & & & & $<0.001^{\mathrm{a}}$ \\
\hline Group A:Group C & & & & $<0.001^{\mathrm{a}}$ \\
\hline DMS (mm) & $2.4 \pm 0.5$ & $4.1 \pm 0.7$ & $5.8 \pm 0.6$ & $<0.001^{\mathrm{a}}$ \\
\hline DMCR (mm) & $5.22 \pm 0.60$ & $5.00 \pm 0.59$ & $4.77 \pm 0.60$ & 0.113 \\
\hline DMPE widening & & & & $0.001^{\mathrm{b}}$ \\
\hline No & $16(69.6)$ & $24(100)$ & $13(100)$ & \\
\hline Yes & $7(30.4)$ & 0 & 0 & \\
\hline \multicolumn{5}{|l|}{ Ectropion } \\
\hline No & $23(100)$ & $24(100)$ & $13(100)$ & \\
\hline Yes & 0 & 0 & 0 & \\
\hline Lateral extension scar & & & & 0.795 \\
\hline No & $19(82.6)$ & 21 (87.5) & $12(92.3)$ & \\
\hline Yes & $4(17.4)$ & $3(12.5)$ & $1(7.7)$ & \\
\hline Follow-up (mo) & $8.09 \pm 2.92$ & $9.25 \pm 3.05$ & $7.85 \pm 2.88$ & 0.298 \\
\hline
\end{tabular}

Values are presented as mean $\pm S D$ or number (\%). Group $A, \geq 2 \mathrm{~mm}$ above the lower eyelid crease or ridge; group $B$, within lower eyelid crease or ridge to $2 \mathrm{~mm}$ above the lower eyelid crease or ridge; group $\mathrm{C}$, within lower eyelid crease or ridge to $2 \mathrm{~mm}$ below the lower eyelid crease or ridge.

POSAS, Patient and Observer Scar Assessment Scale; DMS, distance between the lower eyelid margin and the scar; DMCR, distance between the lower eyelid margin and the lower eyelid crease or ridge; DMPE, distance between the margins of the peripheral pupil and the lower eyelid.

a)Analysis of variance or Fisher exact test for count data and Scheffé's post-hoc test, statistically significant at $p<0.05$. infection or facial asymmetry postoperatively.

Of the 60 patients included in this study, 35 patients were followed up at 6 months after surgery and 25 patients were followed up at 12 months after surgery. According to the location of the scar, 23 patients were classified as group A, 24 patients as group $\mathrm{B}$, and 13 patients as group $\mathrm{C}$ (Fig. 2).

\section{Summary of patient data}

In all patients, the lower eyelid crease or ridge could be identified. The average age was $43.2 \pm 17.2$ years in group $\mathrm{A}$, $41.8 \pm 13.8$ years in group $B$, and $34.1 \pm 17.3$ years in group $C$, but these differences among the three groups were not statistically significant. Likewise, there were no significant differences in the fracture site or fracture type among groups. The average score on the POSAS scar scale was significantly lower in group $B$ than in the other two groups. According to the post-hoc analysis using Scheffés test, the POSAS scores of groups A and $B$ were significantly lower than that of group C. However, there was no statistically significant difference between group A and group B. The DMS was evenly distributed, with a minimum of $2 \mathrm{~mm}$ and a maximum of $7 \mathrm{~mm}$. The DMCR of the operated eye was $5.22 \pm 0.60 \mathrm{~mm}$ in group $\mathrm{A}, 5.00 \pm 0.59 \mathrm{~mm}$ in group $\mathrm{B}$, and $4.77 \pm 0.60 \mathrm{~mm}$ in group $\mathrm{C}$, but these differences among the three groups were not statistically significant (Table 1). Additionally, there were no significant differences in the DMCR of both eyes between the groups (Table 2). On the preoperative snap back test and lid distraction test, none of the patients showed ectropion or asymmetry of the lower eyelid. DMPE widening was observed in seven of the 23 (30.4\%) patients in group $\mathrm{A}$, but in none of the patients of group $\mathrm{B}$ or group $\mathrm{C}$. None of the 60 patients had postoperative ectropion. A lateral extension scar was observed in four of the $23(17.4 \%)$ patients

Table 2. DMCR of both eyes in each group

\begin{tabular}{lcc}
\hline Group & DMCR $(\mathrm{mm})$, mean $\pm S D$ & $p$-value \\
\hline Group A & 0.328 \\
Fractured side & $5.22 \pm 0.60$ & \\
Normal side & $5.26 \pm 0.62$ & 0.082 \\
Group B & \\
Fractured side & $5.00 \pm 0.59$ & \\
Normal side & $5.13 \pm 0.68$ & 0.337 \\
Group C & \\
Fractured side & $4.77 \pm 0.60$ & \\
Normal side & $4.85 \pm 0.69$ & \\
\hline
\end{tabular}

Group $\mathrm{A}, \geq 2 \mathrm{~mm}$ above the lower eyelid crease or ridge; group $\mathrm{B}$, within lower eyelid crease or ridge to $2 \mathrm{~mm}$ above the lower eyelid crease or ridge; group $\mathrm{C}$, within lower eyelid crease or ridge to $2 \mathrm{~mm}$ below the lower eyelid crease or ridge. DMCR, distance between the lower eyelid margin and the lower eyelid crease or ridge.

a)Paired $t$-test, statistically significant at $p<0.05$. 
in group $\mathrm{A}$, three of the $24(12.5 \%)$ patients in group $\mathrm{B}$, and one of the 13 (7.7\%) patients in group C (Table 1).

\section{Descriptive analysis of POSAS scores}

In the POSAS, the total score of observer components and patient components had a similar trend. The average score of the observer components scale was significantly lower in group B than in the other two groups. In the observer components, relief and pliability showed significant differences among the three groups. Relief was defined as the extent to which surface irregularities were present, and pliability referred to the suppleness of the scar tested by wrinkling the scar. The average score of the patient components scale was significantly lower in group $B$ than in the other two groups. In the patient components, color difference, thickness, and scar irregularity showed significant differences among the three groups (Table 3).

\section{Analysis of the relationship between the POSAS score and the DMS}

The relationship between the POSAS score and the DMS is shown in Table 4. The average POSAS score was $22.7 \pm 8.0$ in group A, $20.9 \pm 2.4$ in group B, and $32.5 \pm 4.1$ in group C. In order to confirm the relationship between these two factors (the

Table 3. POSAS score in each group

\begin{tabular}{|c|c|c|c|c|}
\hline Variable & $\begin{array}{l}\text { Group A } \\
(n=23)\end{array}$ & $\begin{array}{l}\text { Group B } \\
(n=24)\end{array}$ & $\begin{array}{l}\text { Group C } \\
(n=13)\end{array}$ & $p$-value \\
\hline \multicolumn{5}{|l|}{ Observer component } \\
\hline Vascularity & $1.3 \pm 0.5$ & $1.3 \pm 0.5$ & $1.5 \pm 0.5$ & 0.328 \\
\hline Pigmentation & $1.1 \pm 0.3$ & $1.0 \pm 0.2$ & $1.3 \pm 0.5$ & 0.249 \\
\hline Thickness & $1.7 \pm 0.8$ & $1.6 \pm 0.5$ & $1.7 \pm 0.5$ & 0.930 \\
\hline Relief & $1.3 \pm 0.4$ & $1.1 \pm 0.3$ & $2.0 \pm 0.4$ & $<0.001^{\mathrm{a})}$ \\
\hline Pliability & $1.3 \pm 0.5$ & $1.2 \pm 0.4$ & $1.8 \pm 0.4$ & $0.006^{\mathrm{a})}$ \\
\hline Surface area & $1.5 \pm 0.7$ & $1.3 \pm 0.5$ & $1.6 \pm 0.5$ & 0.690 \\
\hline Total score observer scar scale & $8.2 \pm 2.3$ & $7.5 \pm 1.2$ & $10.0 \pm 1.2$ & $0.025^{\mathrm{a})}$ \\
\hline \multicolumn{5}{|l|}{ Patient component } \\
\hline Is the scar painful? & $1.3 \pm 0.5$ & $1.3 \pm 0.6$ & $1.6 \pm 0.7$ & 0.150 \\
\hline Is the scar itching? & $1.8 \pm 0.7$ & $1.5 \pm 0.6$ & $1.8 \pm 0.8$ & 0.628 \\
\hline $\begin{array}{l}\text { Is the color of the scar } \\
\text { different? }\end{array}$ & $2.2 \pm 0.7$ & $2.4 \pm 0.7$ & $3.9 \pm 1.1$ & $<0.001^{\text {a) }}$ \\
\hline Is the scar more stiff & $3.3 \pm 2.0$ & $2.8 \pm 0.8$ & $4.2 \pm 2.0$ & 0.270 \\
\hline $\begin{array}{l}\text { Is the thickness of the scar } \\
\text { different? }\end{array}$ & $2.5 \pm 1.1$ & $2.8 \pm 0.7$ & $5.7 \pm 1.3$ & $<0.001^{\text {a) }}$ \\
\hline Is the scar irregular? & $3.3 \pm 2.3$ & $2.6 \pm 0.8$ & $5.3 \pm 1.7$ & $0.017^{a)}$ \\
\hline Total score patient scar scale & $14.5 \pm 6.0$ & $13.4 \pm 2.0$ & $22.5 \pm 3.7$ & $<0.001^{\mathrm{a})}$ \\
\hline \multicolumn{5}{|c|}{$\begin{array}{l}\text { Values are presented as mean } \pm S D \text {. Group } A, \geq 2 \mathrm{~mm} \text { above the lower eyelid } \\
\text { crease or ridge; group B, within lower eyelid crease or ridge to } 2 \mathrm{~mm} \text { above the } \\
\text { lower eyelid crease or ridge; group C, within lower eyelid crease or ridge to } 2 \mathrm{~mm} \\
\text { below the lower eyelid crease or ridge. } \\
\text { POSAS, Patient and Observer Scar Assessment Scale. } \\
\text { a)Analysis of variance, statistically significant at } p<0.05 \text {. }\end{array}$} \\
\hline
\end{tabular}

POSAS score and DMS) and to exclude the influence of other factors that could affect the POSAS score, data were adjusted for age, follow-up period, and the presence of a lateral extension scar. A statistical analysis was performed on the effect of the fracture type and the thickness of the plate used in the orbital rim or orbital floor. Patients who underwent surgery for inferior orbital wall fractures were compared to those who underwent surgery for both tripod and inferior orbital wall fractures, revealing that differences in the fracture type and the plate used did not show statistically significant association with the POSAS score. Follow-up time was also not significantly associated with the POSAS score. Even after adjustment for all of the above factors, a significant positive correlation between the POSAS and the DMS was found, following the equation POSAS= $2.4 \times$ DMS (Table 4$)$.

\section{Analysis of the relationship between the DMS and DMPE widening}

Table 5 shows the results of logistic regression analysis of the correlation between the DMS and the presence of DMPE widening. Only seven of the patients in group A had DMPE widening. The effects of age, follow-up time, and the presence of lateral extension were evaluated. The risk of DMPE widening was found to exhibit a significant positive relationship with age, while follow-up time did not have a significant influence on the risk of DMPE widening. Upon adjusting for all of the above

Table 4. POSAS scores of postoperative scars and DMS

\begin{tabular}{lcc}
\hline Variable & Coefficient $(95 \% \mathrm{Cl})$ & $p$-value \\
\hline DMS & $2.40(1.15$ to 3.66$)$ & $<0.001^{\mathrm{a})}$ \\
Age & $0.07(-0.04$ to 0.18$)$ & 0.186 \\
Follow-up & $-0.12(-0.68$ to 0.44$)$ & 0.674 \\
Lateral extension scar & $2.50(-2.62$ to 7.62$)$ & 0.332 \\
Inferior orbital wall fracture & $-1.32(-5.57$ to 2.92$)$ & 0.534 \\
$\begin{array}{l}\text { Tripod fracture and inferior orbital wall } \\
\text { fracture }\end{array}$ & $-2.18(-8.04$ to 3.68$)$ & 0.459 \\
\hline
\end{tabular}

POSAS, Patient and Observer Scar Assessment Scale; DMS, distance between the lower eyelid margin and the scar; $\mathrm{Cl}$, confidence interval.

a) Linear regression analysis, statistically significant at $p<0.05$.

Table 5. DMS and DMPE widening

\begin{tabular}{lll}
\hline & OR $(95 \% \mathrm{Cl})$ & $p$-value \\
\hline DMS & $0.14(0.01-0.54)$ & $0.029^{\mathrm{a})}$ \\
Age & $1.13(1.03-1.33)$ & $0.048^{\mathrm{a})}$ \\
Follow-up & $1.20(0.79-1.93)$ & 0.392 \\
Lateral extension scar & $0.75(0.04-12.01)$ & 0.841 \\
\hline
\end{tabular}

DMS, distance between the lower eyelid margin and the scar; DMPE, distance between the margins of the peripheral pupil and the lower eyelid; $\mathrm{OR}$, odds ratio; $\mathrm{Cl}$, confidence interval.

a)Logistic regression analysis, statistically significant at $p<0.05$. 
Table 6. Data summary of patients without DMPE widening

\begin{tabular}{|c|c|c|c|c|}
\hline Variable & $\begin{array}{l}\text { Group A } \\
(n=16)\end{array}$ & $\begin{array}{l}\text { Group B } \\
(n=24)\end{array}$ & $\begin{array}{l}\text { Group C } \\
(n=13)\end{array}$ & $p$-value \\
\hline Age (yr) & $37.2 \pm 16.8$ & $41.8 \pm 13.8$ & $34.1 \pm 17.3$ & 0.660 \\
\hline Fracture type & & & & 0.541 \\
\hline Tripod fracture & $10(62.5)$ & $14(58.3)$ & $9(69.2)$ & \\
\hline Inferior orbital wall fracture & $3(18.8)$ & $8(33.3)$ & $4(30.8)$ & \\
\hline Both & $3(18.8)$ & $2(8.3)$ & 0 & \\
\hline Fracture site & & & & 0.966 \\
\hline Medial & $3(18.8)$ & $5(20.8)$ & $4(30.8)$ & \\
\hline Central & $10(62.5)$ & $15(62.5)$ & $7(53.8)$ & \\
\hline Lateral & $3(18.8)$ & $4(16.7)$ & $2(15.4)$ & \\
\hline POSAS & $17.7 \pm 2.2$ & $20.9 \pm 2.4$ & $32.5 \pm 4.1$ & $<0.001^{\mathrm{a}}$ \\
\hline Group A:Group B & & & & $0.004^{\mathrm{a}}$ \\
\hline Group B:Group C & & & & $<0.001^{\mathrm{a}}$ \\
\hline Group A:Group C & & & & $<0.001^{\mathrm{a}}$ \\
\hline DMS (mm) & $2.5 \pm 0.5$ & $4.1 \pm 0.7$ & $5.8 \pm 0.6$ & $<0.001^{\mathrm{a}}$ \\
\hline DMCR (mm) & $5.12 \pm 0.06$ & $5.00 \pm 0.59$ & $4.77 \pm 0.60$ & 0.308 \\
\hline \multicolumn{5}{|l|}{ DMPE widening } \\
\hline No & $16(100)$ & $24(100)$ & $13(100)$ & \\
\hline Yes & 0 & 0 & 0 & \\
\hline \multicolumn{5}{|l|}{ Ectropion } \\
\hline No & $16(100)$ & $24(100)$ & $13(100)$ & \\
\hline Yes & 0 & 0 & 0 & \\
\hline Lateral extension scar & & & & 1.000 \\
\hline No & $14(87.5)$ & $21(87.5)$ & $12(92.3)$ & \\
\hline Yes & $2(12.5)$ & $3(12.5)$ & $1(7.7)$ & \\
\hline Follow-up (mo) & $7.88 \pm 2.87$ & $9.25 \pm 3.05$ & $7.85 \pm 2.88$ & 0.263 \\
\hline
\end{tabular}

Values are presented as mean $\pm S D$ or number (\%). Group $A, \geq 2 \mathrm{~mm}$ above the lower eyelid crease or ridge; group B, within lower eyelid crease or ridge to $2 \mathrm{~mm}$ above the lower eyelid crease or ridge; group $\mathrm{C}$, within lower eyelid crease or ridge to $2 \mathrm{~mm}$ below the lower eyelid crease or ridge.

DMPE, distance between the margins of the peripheral pupil and the lower eyelid; POSAS, Patient and Observer Scar Assessment Scale; DMS, distance between the lower eyelid margin and the scar; DMCR, distance between the lower eyelid margin and the lower eyelid crease or ridge.

a)Analysis of variance or Fisher exact test for count data and Scheffé's post-hoc test, statistically significant at $p<0.05$

factors, the risk of DMPE widening was found to increase as the DMS decreased (Table 5).

\section{POSAS score between groups without DMPE widening}

In a subgroup analysis of 53 patients without DMPE widening, the POSAS score was lower in group A than in group B, and the DMS showed a clear positive correlation with the POSAS score (Tables 6 and 7).

\section{DISCUSSION}

\section{Approach methods}

The selection of an appropriate lower eyelid approach for orbit-
Table 7. POSAS scores of postoperative scars and DMS (in patients without DMPE widening)

\begin{tabular}{lcc}
\hline & Coefficient (95\% Cl) & $p$-value \\
\hline DMS & $3.97(3.20$ to 4.74$)$ & $<0.001^{\text {a) }}$ \\
Age & $-0.04(-0.11$ to 0.02) & 0.207 \\
Follow-up & $-0.12(-0.46$ to 0.22) & 0.407 \\
Lateral extension scar & $0.39(-2.87$ to 3.65$)$ & 0.810 \\
Inferior orbital wall fracture & $-1.05(-3.47$ to 1.37$)$ & 0.387 \\
$\begin{array}{l}\text { Tripod fracture and inferior orbital wall } \\
\text { fracture }\end{array}$ & $-1.76(-5.40$ to 1.87$)$ & 0.334 \\
\hline
\end{tabular}

POSAS, Patient and Observer Scar Assessment Scale; DMS, distance between the lower eyelid margin and the scar; DMPE, distance between the margins of the peripheral pupil and the lower eyelid; $\mathrm{Cl}$, confidence interval.

a) Linear regression analysis, statistically significant at $p<0.05$.

al wall reconstruction is very important. The lower eyelid approach may cause postoperative complications such as scleral show, ectropion, entropion, and canthal malposition, as well as postoperative scarring [13]. These complications induce eye discomfort and dry eye syndrome, as well as cosmetic complaints, and increase the duration and costs of treatment [14].

The inferior orbital wall can be accessed by a transconjunctival or a transcutaneous approach. Since the transconjunctival approach does not involve a skin excision, it does not leave a postoperative external scar and the risk of ectropion is very low. However, if extensive exposure of the orbital rim or orbital floor is required, a lateral canthotomy should be additionally performed. A lateral canthotomy increases the likelihood of complications such as entropion and canthal malposition [15]. Depending on the incision, the transcutaneous approach can be classified into three types: subciliary, subtarsal, and infraorbital. It can also be divided into skin flap, non-stepped skin-muscle flap, and stepped skin-muscle flap methods, depending on the dissection technique $[5,6]$.

Extensive efforts have been made to reduce the incidence of side effects using the transcutaneous approach. Compared to subtarsal or infraorbital incisions, subciliary incisions cause less conspicuous scars and less postoperative edema; however, they increase the risk of scleral show or ectropion [15]. Lower eyelid malposition such as ectropion and scleral show is a common side effect of the transcutaneous approach. It is thought to occur as a result of the weakening of the bond between the tarsal plate and the orbicularis muscle, according to the surgical approach, the contractions occurring in the scar formation process, or decreased muscle tone when the zygomatic branch of the facial nerve innervating the orbicularis oculi muscle is damaged during dissection $[13,16]$. When the stepped skin-muscle flap method is used, damage to the pretarsal fibers of the orbicularis muscle attached to the tarsal plate can be prevented and 
the lower eyelid is in contact with the eyeball without malpositioning, thereby reducing the risk of ectropion [5].

\section{Lower eyelid crease and ridge}

In Caucasians, the lower eyelid crease has a structure similar to that of the upper eyelid crease. The upper eyelid crease is formed according to the shape of the levator aponeurosis, which is attached to the upper margin of the tarsal plate and bonded with the skin. In the lower eyelid, similar to the levator aponeurosis of the upper eyelid, the capsulopalpebral fascia binds to the lower margin of the lower eyelid tarsus. It penetrates the orbicularis oculi muscle at the site where this capsulopalpebral fascia joins the tarsus and partially attaches to the skin to form a lower eyelid crease. The capsulopalpebral fascia binds extensively to the skin; therefore, the lower eyelid is thinner, and the lower eyelid crease is concave.

In young Asians, however, the capsulopalpebral fascia and the orbital septum are joined in the upper part closer to the tarsal plate, and there is almost no connection between the fascia and the skin. The orbital fat is projected forward and upward and the orbicularis oculi and skin covering the surface are affected [9-11]. Therefore, the thin pretarsal skin and thick preseptal skin form a natural fold at a level similar to that of the lower eyelid crease in Caucasians, resulting in a ridge-shaped line. The authors named this fold the "lower eyelid ridge" (Fig. 4). With aging, this lower eyelid ridge in young Asians changes to a crease-shaped fold, as the lowering of orbital fat and soft tissue progresses.

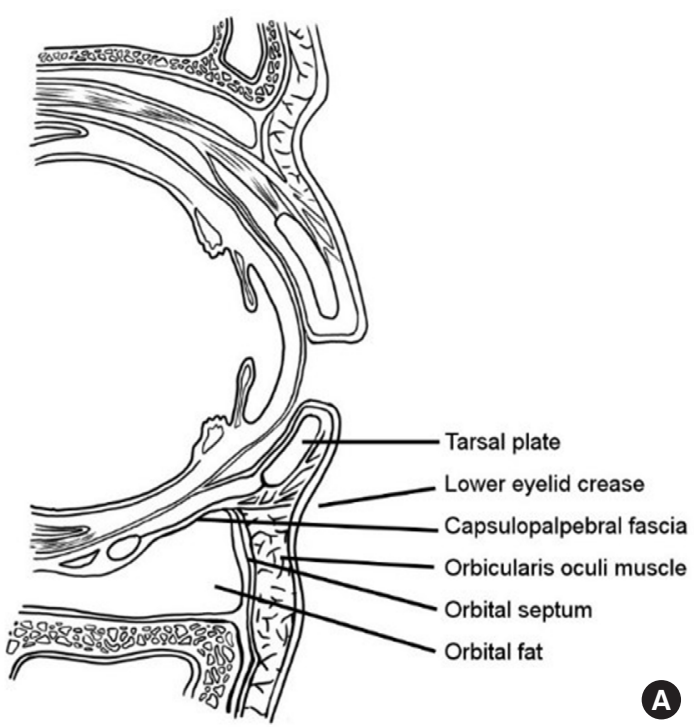

The lower eyelid crease or ridge was located $5 \mathrm{~mm}$ below the lower lid margin on average, and this distance did not show any significant between group differences in this study (Table 1). According to Kim and Hwang [17], the tarsal plate height of the lower eyelid was similar between Caucasians $(4-5 \mathrm{~mm})$ and Asians $(4.6 \mathrm{~mm})$. The average height of the lower eyelid tarsal plate and the average DMCR were similar. Therefore, the authors believe that based on the anatomical structure described above, the lower eyelid crease or ridge can be used as a surface landmark that indicates the location of the lower margin of the lower eyelid tarsal plate.

\section{Lower eyelid contracture}

To evaluate the occurrence of scleral show due to scar contracture, the distance from the peripheral margin of the iris to the lower lid margin should be checked. However, in Asians, the lower eyelid usually covers the lower peripheral margin of the iris to a significant extent. Therefore, the authors compared the DMPE of the operated eye to that of the unoperated eye as an alternative. DMPE widening was defined as a difference in the DMPE of $\geq 1 \mathrm{~mm}$ between the operated and unoperated sides.

The risk of DMPE widening was inversely associated with the DMS, and the POSAS score was positively associated with the DMS. However, in this study, the POSAS score was higher in group $\mathrm{A}$, in which the DMS was shorter than that in group $\mathrm{B}$. As a sensitivity analysis, a subgroup analysis of 53 patients without DMPE widening was performed. In a subgroup study, the POSAS score in group A was lower than that in group B (Tables

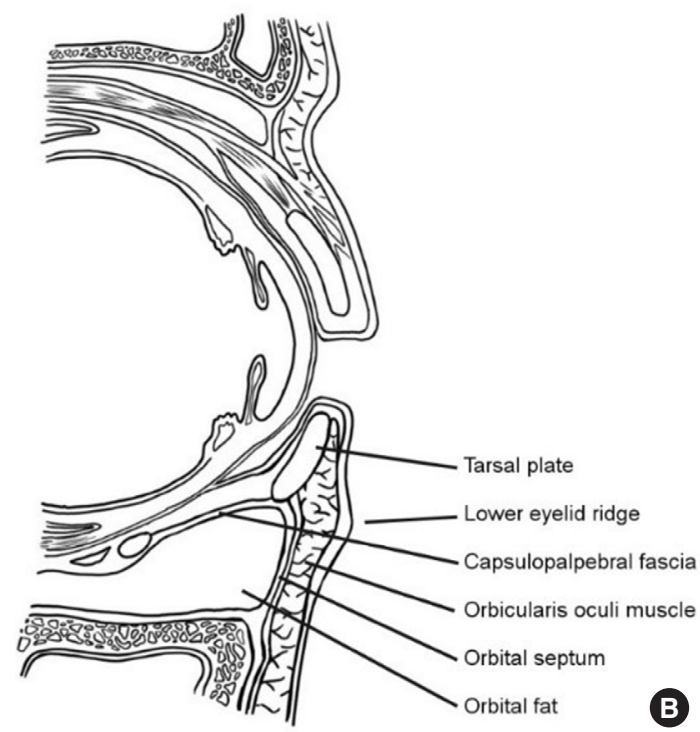

Fig. 4. Lower eyelid crease and ridge. (A) In Caucasians, the lower eyelid crease is formed by the fascial extensions of the capsulopalpebral fascia, which also pass through the orbicularis oculi muscle and insert into the skin. (B) In young Asians, the crease is absent or ambiguous, or is replaced with a transverse ridge due to higher or indistinct septum fusion, anterior and superior orbital fat projection, and overriding of the preseptal orbicularis muscle. 
$6,7)$. In light of this finding, the authors believe that higher levels of dissatisfaction with scars and asymmetry may have led to higher POSAS scores.

\section{Incision line location}

In previous studies, the location of the incision line was determined according to the distance from the lower eyelid margin. However, this method might not be accurate because of preoperative swelling. Instead, in this study, the location of the incision line was estimated on the basis of the location of the postoperative scar. This method accounts for the possibility that the location of the scar could change from that of the incision line with progression of wound healing and scarring. Therefore, determining the location of the incision line based on an analysis of the postoperative scar could have led to distortion. To clarify whether distortion had occurred, the involved lower eyelid was evaluated compared to the normal eyelid on the contralateral side. After the complete resolution of postoperative edema, the DMCR was measured in both eyes. The absence of a remarkable difference between the eyes confirmed that the location of the lower eyelid crease or ridge did not change after surgery (Table 2). Based on these results, the authors concluded that the location of the scar could represent the line of the incision to some degree. The findings of this study indicate that for lower eyelid surgery in Asians, it is preferable to make an incision within $2 \mathrm{~mm}$ above the lower eyelid crease or ridge, considering overall patient satisfaction.

\section{Study limitations}

This study has some limitations including a small sample size and a short follow-up period. In all patients of this study, the lower eyelid crease or ridge could be identified. However, the lower eyelid crease or ridge could not be identified in elderly patients or others whose orbital fat and soft tissue lowering, or lower eyelid wrinkling had progressed. Hence, further studies with a wider range of demographics, and prospective studies using the lower eyelid crease or ridge as a surgical landmark, would help clarify these results.

\section{Summary}

The lower eyelid crease or ridge may be used as a specific anatomical landmark to determine the location of the lower eyelid incision according to the unique characteristics of Asians. When using the transcutaneous approach for inferior orbital wall reconstruction, the optimal incision site is within the lower eyelid crease or ridge to $2 \mathrm{~mm}$ above the lower eyelid crease or ridge.

\section{NOTES}

\section{Conflict of interest}

Kwang Seog Kim and Jae Ha Hwang are editorial board member of the journal but were not involved in the peer reviewer selection, evaluation, or decision process of this article. No other potential conflicts of interest relevant to this article were reported.

\section{Ethical approval}

The study was approved by the Institutional Review Board of Chonnam National University Hospital (IRB No. CNUH-2021342) and performed in accordance with the principles of the Declaration of Helsinki. Written informed consent was obtained.

\section{Patient consent}

The patient provided written informed consent for the publication and the use of his images.

\section{ORCID}

$\begin{array}{ll}\text { Seong Jin Oh } & \text { https://orcid.org/0000-0003-4057-9398 } \\ \text { Kwang Seog Kim } & \text { https://orcid.org/0000-0002-6766-4640 } \\ \text { Jun Ho Choi } & \text { https://orcid.org/0000-0002-4848-517X } \\ \text { Jae Ha Hwang } & \text { https://orcid.org/0000-0001-6992-8067 } \\ \text { Sam Yong Lee } & \text { https://orcid.org/0000-0002-3185-2519 }\end{array}$

Author contribution

Conceptualization: Kwang Seog Kim. Data curation: Seong Jin Oh, Jun Ho Choi. Formal analysis: Jae Ha Hwang, Sam Yong Lee. Methodology: Jae Ha Hwang, Sam Yong Lee. Project administration: Kwang Seog Kim. Writing - original draft: Seong Jin Oh, Kwang Seog Kim, Jun Ho Choi. Writing - review \& editing: Kwang Seog Kim. Investigation: Seong Jin Oh, Jun Ho Choi.

\section{REFERENCES}

1. Lee H, Kim KS, Choi JH, Hwang JH, Lee SY. Trauma severity and mandibular fracture patterns in a regional trauma center. Arch Craniofac Surg 2020;21:294-300.

2. Kim SH, Choi JH, Hwang JH, Kim KS, Lee SY. Surgical indication analysis according to bony defect size in pediatric orbital wall fractures. Arch Craniofac Surg 2020;21:276-82.

3. Cho J, Kim Y, Choi Y. Three-dimensional analysis of facial asymmetry after zygomaticomaxillary complex fracture reduction: a retrospective analysis of 101 East Asian patients. Arch Craniofac Surg 2021;22:148-53.

4. Lee YJ. Orbital floor fracture repair with implants: a retrospec- 
tive study. Arch Craniofac Surg 2021;22:177-82.

5. Rohrich RJ, Janis JE, Adams WP Jr. Subciliary versus subtarsal approaches to orbitozygomatic fractures. Plast Reconstr Surg 2003;111:1708-14.

6. Bronstein JA, Bruce WJ, Bakhos F, Ishaq D, Joyce CJ, Cimino V. Surgical approach to orbital floor fractures: comparing complication rates between subciliary and subconjunctival approaches. Craniomaxillofac Trauma Reconstr 2020;13:45-8.

7. Ridgway EB, Chen C, Colakoglu S, Gautam S, Lee BT. The incidence of lower eyelid malposition after facial fracture repair: a retrospective study and meta-analysis comparing subtarsal, subciliary, and transconjunctival incisions. Plast Reconstr Surg 2009;124:1578-86.

8. Neovius E, Clarliden S, Farnebo F, Lundgren TK. Lower eyelid complications in facial fracture surgery. J Craniofac Surg 2017; 28:391-3.

9. Hawes MJ, Dortzbach RK. The microscopic anatomy of the lower eyelid retractors. Arch Ophthalmol 1982;100:1313-8.

10. Lim WK, Rajendran K, Choo CT. Microscopic anatomy of the lower eyelid in Asians. Ophthalmic Plast Reconstr Surg 2004; 20:207-11.

11. Kakizaki H, Jinsong Z, Zako M, Nakano T, Asamoto K, Miyai- shi $\mathrm{O}$, et al. Microscopic anatomy of Asian lower eyelids. Ophthalmic Plast Reconstr Surg 2006;22:430-3.

12. Kim H, Kim J, Choi J, Jung W. The usefulness of Leukosan SkinLink for simple facial laceration repair in the emergency department. Arch Plast Surg 2015;42:431-7.

13. Fernandes Lopes Morais D’Autilio M, Bottini DJ, Basile E, Lovero S, Sanese G, de Angelis B, et al. Incidence and prevention of cicatricial scleral show in the subciliary access in patients with orbital fractures. J Craniofac Surg 2019;30:1782-6.

14. Kim CH, Choi WY, Son KM, Cheon JS. Prediction of lower eyelid malpositioning after surgical correction of orbital fracture using the subciliary approach through the canthal area and orbital vector analysis. J Craniofac Surg 2020;31:e297-9.

15. Haghighat A, Moaddabi A, Soltani P. Comparison of subciliary, subtarsal and transconjunctival approaches for management of zygomaticoorbital fractures. Br J Med Med Res 2017;20:1-9.

16. Kim YK, Kim JW. Evaluation of subciliary incision used in blowout fracture treatment: pretarsal flattening after lower eyelid surgery. Plast Reconstr Surg 2010;125:1479-84.

17. Kim YS, Hwang K. Shape and height of tarsal plates. J Craniofac Surg 2016;27:496-7. 